Chapter 14

\title{
Aerobic Biodegradation Coupled to \\ Preliminary Ozonation for the Treatment \\ of Model and Real Residual Water
}

\author{
P. Guerra, J. Amacosta, T. Poznyak, S. Siles, \\ A. García and I. Chairez
}

Additional information is available at the end of the chapter

http://dx.doi.org/10.5772/56011

\section{Introduction}

\subsection{Sequence of water treatment methods}

Residual and waste water have become a problem of paramount importance in modern societies [1]. Recently, the number of proposals to solve this issue has incremented importantly [2]. Several methods were proposed since thirty years ago using a wide variety of physical, biological and chemical principles. Biological treatments are cheap and environmentally friendly [3]. Nevertheless, they require a long time to eliminate pollutants and they are limited by the toxicity and initial concentration of the water sample that must be treated [4]. On the other hand, chemical treatments are capable to promote the faster decomposition for a wide range of toxic compounds [5]. Despite this adequate performance to decompose organics dissolved in water, they are hundreds or thousands of times more expensive than pure biological methods [6-11].

\subsection{Ozonation followed by biodegradation}

Just some years ago, the attractive features of both methods (biological and chemical) have attracted attention to develop more advanced schemes to manage more toxic and complex pollutant mixtures [12-13]. Indeed, remarkable results have come from a sequence of treatments (usually called trains of treatments) using the combination of several individual options. Regularly, the treatment trains are using a sequence defined by a physical method followed by the biological scheme and finally, one last chemical process completes the treatment. 
However, this arrangement does not always work efficiently when the initial pollutant mixture has complex composition or they are very toxic. The stage that is usually compromised by this aspect is the biological one [14-15].

Several mixed processes have been recently proposed including a process based on chemical oxidative compounds plus biological based decomposition course [12-18]. Among others, oxygen injected with high pressure, ozonation [13], catalytic and photocatalytic processes and others have been tested to perform the chemical decomposition [16-18]. Most of these treatment methods have important advantages but also have important drawbacks. These problems can be classified into two main areas: the first one contains all economic aspects associated with high cost required to implement these treatments, the second one includes all troubles associated to the resources needed to complete the transformation from very toxic compounds to simpler ones that can be considered as no toxicity and no hazardous [19]. Nevertheless, these drawbacks may be solved by biologically based treatments.

Nowadays, a different way of thinking has emerged to improve the efficiency of waste and residual water: changing the order of treatments to include a chemical pre-treatment before the biological process. The idea is to reduce the complexity as well as the toxicity of the organic mixture of chemical methods. Theoretically, this condition must have a positive effect on the microorganism's efficiency to decompose the simpler and less toxic organics.

Beltran et al. [13] reported that combined ozonation and aerobic treatment increased the removal efficiency from $82 \%$ or $76 \%$ for the COD or the total phenolic content, respectively. Benitez et al. [20] demonstrated the COD removal for wine vinasses containing organic matter and aromatic compounds was enhanced (from $27.7 \%$ to $39.3 \%$ ), when the combined ozonation and biological process was used. Aparicio et al. [21] reported the use of combined wastewater treatment set up in a resin-producing factory. After biological treatment of the ozonated effluent, the organic carbon and nitrogen removal was increased from 27 to $97 \%$ and from 27 to $80 \%$, respectively.

The possible benefits coming from the combination of pre-treatment with ozone and a sequential biodegradation are almost evident; however, there are still several questions about this procedure. For example, what time is adequate to move the organics mixture from the ozonation reactor to the biological one or what conditions should be set-up for both reactions still remain as open problems. Another important issue that must be explained is what conditions must fulfill the microorganism strains to handle the pollutant mixture produced by the preliminary ozonation. This is an important aspect conditioned to the composition of the mixture supplied to microorganisms that can modify the organics elimination by biodegradation. Moreover, there is just a few of works describing what type of microorganisms is responsible for the elimination of residual compounds after ozonation [22]. In recent reports, [23-29] catechol, hydroquinone and several low weight organic acids have been recognized like the main byproducts obtained after ozonation of phenol and its chlorinated derivatives. Nevertheless, what relative concentration of each byproduct is the most adequate to construct the combined process including ozonation and biological reaction has not been determined yet. 


\subsection{Phenol and its chlorinated derivatives as artificial wastewater}

Phenol and its chlorinated derivatives are simple examples of how the biodegradation can be efficient or not for closely related pollutants [26, 27]. It has been broadly reported the efficient decomposition of phenol by biological strands. Many methods of eliminating phenol and its derivatives using chemical and biological systems have been studied [23-24]. Biological systems are environmentally friendly, low-cost technologies that can be successfully used to remove phenols by using different microbial strains but with pure cultures.

However, when any chlorophenol is exposed to the same microorganisms, the toxicity of this compound reduces the decomposition efficiency to 20 or $30 \%$ compared to the same conditions observed in phenol treatment.

On the other hand, most of advanced oxidation processes can remove the chlorine atom from the chlorophenols in few minutes or even seconds [5]. Therefore, the possible sequential treatment based on ozone followed by biodegradation can use the advantages offered by this couple of water treatment schemes. Indeed, phenol's ozonation generates simpler organic acids that can be assimilated by microorganisms [14-15].

\subsection{Lignin and its derivatives as real wastewater}

A more complex situation arises when the pollutants in residual water are toxic and also with complex structure. As an example, pulp and paper industry wastewater mainly contains lignin and its derivatives (chlorinated phenolic compounds, resin acids, dioxins and dioxin-like compounds and many others) [29-32].

Lignin is a three-dimensional biopolymer which confers the resistance to stress, protects the plant from the microbial enzymatic hydrolysis and also acts as a binder of the fibers of cellulose and hemicellulose in the wood [33,34]. Is formed by the coupling of three monolignols (paracoumaryl alcohol, coniferyl alcohol and sinapyl alcohol) and with some functional groups $\left(\mathrm{ROH}, \phi \mathrm{OH}, \mathrm{ROMe}, \mathrm{RR}^{\prime} \mathrm{C}=\mathrm{O}, \mathrm{RCOOH}, \mathrm{RSO}_{3} \mathrm{R}^{\prime}\right.$, etc.). The extractable products like fatty acids, phenols, terpenes, steroids, waxes, tannins and resinic acids, also found in the wastewaters, confer the physicochemical properties of each plant such as color, smell, strength, hardness [37-39].

Lignin and its derivatives have shown to be a very complex, toxic mixture with mutagenic and teratogenicity activities. Biological treatment of wastewater with these residuals has partial efficiency [39-41].

\subsection{Motivation and contribution of this study}

In this chapter, a combined method to treat residual water is proposed. The treatment is based on the preliminary action of ozone followed by the biological treatment using a microorganism consortium. Two water samples were used to evaluate the combined treatment: a model mixture of chlorophenols and residual water obtained from the paper industry after the bleaching step from the Kraft process. This selection was done to illustrate the efficiency of the combined process using ozone and biodegradation in a row. 


\section{Materials and methods}

\subsection{Model and real residual water}

Model solutions were artificially prepared with 4-Chlorophenol (4-CPh) or 2,4-Dichlorophenol (2,4-DCPh) (120 mg/L) as model solutions. All these chemical products have $99 \%$ purity.

For the real residual water, the sample was obtained from a Kraft process in the bleaching step; collected at $4{ }^{\circ} \mathrm{C}$ and sterilized in autoclave under the temperature of $121^{\circ} \mathrm{C}$ and a pressure of 15 pounds. The mixture was characterized by simple analytical methods based on UV/VIS spectroscopy.

These two polluted water samples were treated by ozonation, aerobic biodegradation and the combination of both processes (ozonation followed by biodegradation). The biodegradation was developed using a microbial consortium acclimated to the particular composition of carbon source remaining in the reactor after/before ozonation [42-44].

\subsection{Ozonation procedure}

The ozonation treatment was carried out in a semi-batch glass reactor $(250 \mathrm{~mL})$. Ozone concentration at the reactor's input was $30 \mathrm{mg} / \mathrm{L}$. The maximum ozonation time was $60 \mathrm{~min}$ for both the real and artificial wastewater. The ozone/oxygen mixture was injected through a ceramic porous at the inferior part of the reactor with a flow of $0.5 \mathrm{~L} / \mathrm{min}$. The ozone was produced by the ozone generator HTU500G 'G' (corona discharge type, "AZCO” INDUSTRIES LIMITED, Canada). The Ozone Analyzer BMT 963 "S” (BMT Messtechnik, Berlin) provides the ozone detection in the gas phase at the reactor output. This information was used to perform the ozone monitoring, to control the ozonation degree and to study the ozone decomposition. The ozone concentration was sampled by a data acquisition system implemented in a regular computer (Figure 1).

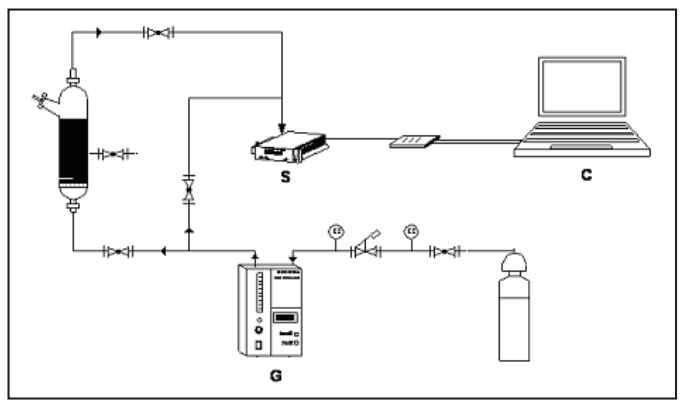

Figure 1. Simple scheme of the ozonation setup including the reactor where the ozonation is carried out. The ozone concentration produced in $(\mathrm{G})$ is monitored in the UV sensor (S). A data acquisition board is connected to a personal computer to register the ozone concentration. 
The ozonation of was carried out using two different pHs: 7 (for model and real water) and 12 (only model solution). Ozonation of residual water samples was carried out for diluted solutions (1:10). This change promotes the reduction of reaction time and helped to decrease foaming [16].

\subsection{Microbial culture, mineral media}

For the biological treatment, different microbial consortia were cultivated and acclimated during 6 months (by a fill-and-draw procedure) to the specific carbon source (model chlorophenols and real water solutions with and without previous ozonation).

The mineral media used for all the experiments contains $(\mathrm{g} / \mathrm{L}): 3.0\left(\mathrm{NH}_{4}\right)_{2} \mathrm{SO} 4,0.6 \mathrm{KH}_{2} \mathrm{PO}_{4}, 2.4$ $\mathrm{K}_{2} \mathrm{HPO}_{4}, 1.5 \mathrm{MgSO}_{4} \bullet 7 \mathrm{H} 2 \mathrm{O}, 0.15 \mathrm{CaSO}_{4}$, and $0.03 \mathrm{FeSO}_{4}$. A mixed microbial culture from a biofilter used to remove aromatic compounds and gasoline vapors (Dr Revah's Laboratory, Universidad Autonoma Metropolitana Iztapalapa, Mexico) was independently adapted for three months to phenol (100 mg/L) and to a mixture of oxalic and formic acids (100 mg/L each) in mineral media.

The mixed culture was cultivated in an Erlenmeyer flask of $1 \mathrm{~L}$ with $500 \mathrm{~mL}$ of mineral media. These compositions were inoculated with $50 \mathrm{~mL}$ of the microbial mixture. Reactors were kept at ambient temperature and shaken in an orbital shaker at 200 RPM. The mineral media was also kept invariable for these experiments. In all the studied samples, the biomass amount and the organic degradation as well were periodically measured in triplicate.

The cultures were harvested between 24 and $30 \mathrm{~h}$, corresponding to the exponential growth phase and then used for the model and real water treatment (phenol and chlorophenols mainly) and their corresponding ozonation products (catechol, hydroquinone and organic acids mainly) [46].

For the biological treatment, different microbial consortia (set of several microorganisms with different species) were cultivated and acclimated during 6 months (by a fill-and-draw procedure) to the specific carbon source (model chlorophenols and real water solutions with or without previous ozonation). In this study, the biological media is composed of a complex consortium of the microbial population previously identified [22] by the extraction of DNA samples using an Easy-DNATM Kit (Invitrogen, USA) [44].

Inoculums of the corresponding microbial consortium were added into the batch reactor containing the model solution or the real water (with or without previous ozonation). Reactors were kept at ambient temperature and shaken in an orbital shaker at 200 RPM. Chlorophenolsenols (from model solution) and real water's components concentration, as well as ozonation products concentration in reactors and the biomass amount were periodically measured by triplicate.

\subsection{Analytical methods}

Several analytical methods were used in order to characterize, identify and quantify the samples. UV Spectroscopy (Lambda 2S, Perkin Elmer) was used for monitoring the global 
behavior of ozonation ( $\lambda=260$ and $210 \mathrm{~nm}$ ) and biodegradation $(\lambda=210 \mathrm{~nm})$ of real water as well as the microbial growth (OD600).

The control of chlorophenols or the components of real water decomposition, as well as the intermediates and final products formed in the ozonation step was made by high performance liquid chromatography (HPLC), (Series 200, Perkin Elmer) equipped with UV-VIS detector series 200. Two wavelengths were periodically monitored (210 $\mathrm{nm}$ and $270 \mathrm{~nm}$ ). Analytical details are shown in Table 1.

\begin{tabular}{|c|c|c|}
\hline \multirow{2}{*}{ Analysis conditions } & \multicolumn{2}{|c|}{ Compounds } \\
\hline & Phenols & Organic acids and real water \\
\hline Column & $\begin{array}{c}\text { Platinum C-18 (Alltech), } 250 x \\
4.6 \mathrm{~mm}\end{array}$ & Prevail Organic Acid (Grace), $150 \times 4.6 \mathrm{~mm}$ \\
\hline Mobile Phase & 60:40 (water : methanol) & $\begin{array}{c}\mathrm{KH}_{2} \mathrm{PO}_{4} 25 \mathrm{mMol} \text { in water } \\
(\mathrm{pH}=2.5)\end{array}$ \\
\hline$\lambda(\mathrm{nm})$ & & 210 \\
\hline Flow rate & & $1 \mathrm{~mL} / \mathrm{min}$ \\
\hline Sample volume & & $30 \mu \mathrm{L}$ \\
\hline
\end{tabular}

Table 1. HPLC analysis conditions

The study was made on raw material and samples of study, both in the stage of ozonation of biodegradation. Identification and qualitative determinations were made taking into account the retention times of components and the quantitative analysis by integration of signals, in relation to the corresponding calibration curve.

\section{Results}

For both kind of waters, model solution or real water, three processes were evaluated: ozonation, biodegradation (without ozonation) and the combined treatment (ozonation followed by biodegradation). Those are described below.

\subsection{Ozonation}

\subsubsection{Model solution preliminary ozonation}

Chlorophenols (CPhs) decomposition was faster at $\mathrm{pH} 12$ (8 and 5 minutes) than $\mathrm{pH} 7$ (15 and 8 minutes) for 4 - $\mathrm{CPh}$ and 2,4-DCPh, respectively. Some by-products like catechol, hydroquinone, oxalic and formic acids were formed. All these are some of the products identified in CPhs ozonation [5]. Besides, some other ones were observed but they could not be identified, however, they were monitored by HPLC and referred as non- identified phenolic compounds 
and organic acids. During ozonation, both identified and non-identified phenolic compounds were rapidly decomposed, while oxalic and formic acids were mostly accumulated during the whole reaction period. The maximum concentration detected for the different ozonation conditions were previously published [5]. All these compounds constituted the carbon source for adapted bioprocess applied at the next step. Then, the percentage of CPhs decomposition and the by-products accumulation/decomposition was considered to stop the ozonation and carry out the biodegradation step.

\subsubsection{Real residual water ozonation}

In the case of real residual wastewater ozonation, a significant decrease of organic compounds concentration was followed in the UV spectra (Figure 2). Lignin derivatives was followed in the UV region of $\lambda=260 \mathrm{~nm}$ and organic acids in the region of $\lambda=210 \mathrm{~nm}$.

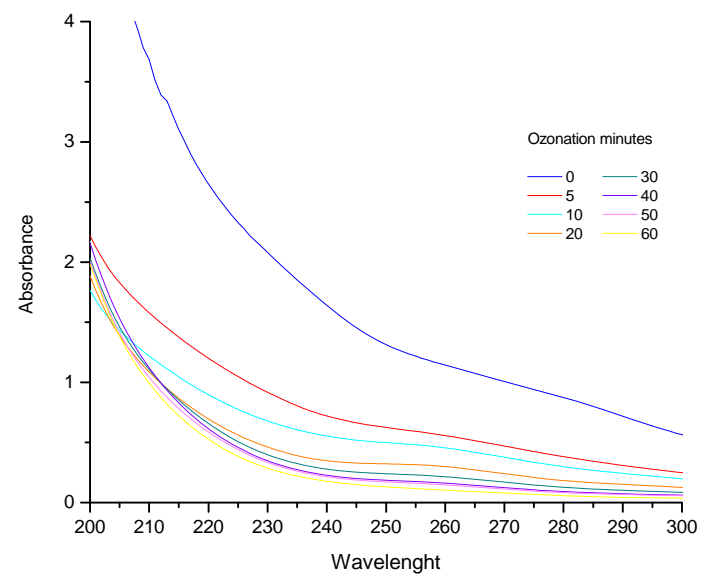

Figure 2. Variation of UV-VIS spectrum in the ozonation process.

A significant decrease in the region of lignin derivatives (94\%) and in the corresponding organic acids $(83 \%)$, which also tends to decrease in absorbance is depicted. At the end of ozonation no longer variation in the UV spectra is observed. Therefore, the susceptible organic matter to be ozonated has been completely reacted with ozone.

The identification of organic compounds in the original sample and also during the ozonation was done by the HPLC technique. The decomposition dynamics during the reaction with ozone was determined. Moreover quantification of accumulated products is gotten. Main byproducts were hydroquinone, catechol and simple organic acids such as maleic acid and several unidentified compounds.

The main recalcitrant accumulated product during ozonation was oxalic acid. Indeed, its presence was observed from the beginning to the end of ozonation. Its relative importance for the sequent biodegradation motivated its quantification by HPLC (Figure 3). 


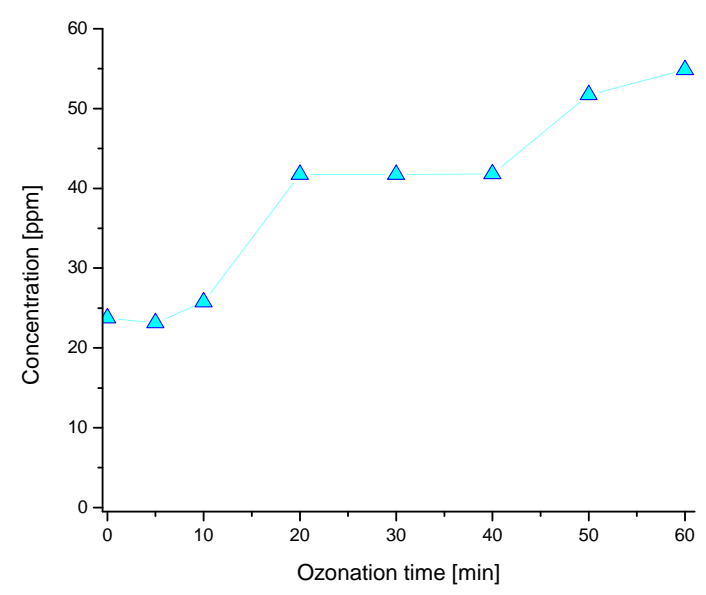

Figure 3. Quantification of oxalic acid during the ozonation of real wastewater.

As it can be seen, oxalic acid was contained in the real residual wastewater before any treatment (23 mg/L), but after the first 30 minutes $43 \mathrm{mg} / \mathrm{L}$ of this acid was detected. After 60 minutes of ozonation the acid concentration was increased up to $55 \mathrm{mg} / \mathrm{L}$.

\subsection{Biodegradation}

\subsubsection{Biological treatment of model solution (without ozonation)}

Poor degradation of CPhs was observed in the case of non ozonated samples $30 \%$ and $40 \%$ for 4-CPh and 2,4-DCPh, respectively) after 10 days. No matter the microorganisms were previously acclimated to $\mathrm{CPhs}$, the initial concentration was toxic enough to inhibit biodegradation, which is one of the biological treatments principal disadvantages. The biodegradation of ozonation products identified during ozonation was also tested. The minority compounds were eliminated obeying the following order of elimination: phenol>catechol $>$ hydroquinone. For final products, a mixture of oxalic and formic acids with a concentration of $100 \mathrm{mg} / \mathrm{L}$ of each one was also tested. Microorganisms were able to eliminate both compounds during two days. These results are very important because they demonstrated that: highly toxic substrates, which cannot be eliminated by bioprocess, but they can be easily degraded by ozone and transform them into several compounds.

These results demonstrated that highly toxic substrates which cannot be eliminated by bioprocess are in the model solution, but they can be easily degraded by ozone and transform them into several compounds. Additionally, an acclimated consortium whit capability to eliminate the ozonation products was produced. So, it was expected that ozonation products were easier to be eliminated by biodegradation than original CPhs. Therefore, one can expect that combined treatment is more efficient than individual ones. 


\subsubsection{Biological treatment of real residual water (without ozonation)}

The real residual water biodegradation showed a similar behavior to model solution one. It showed a partial decomposition of the organic compounds up to $20 \%$ after five days due to the complexity and heterogeneity of this sample without ozonation. Therefore, organic matter degradation is poor (Figure 4).

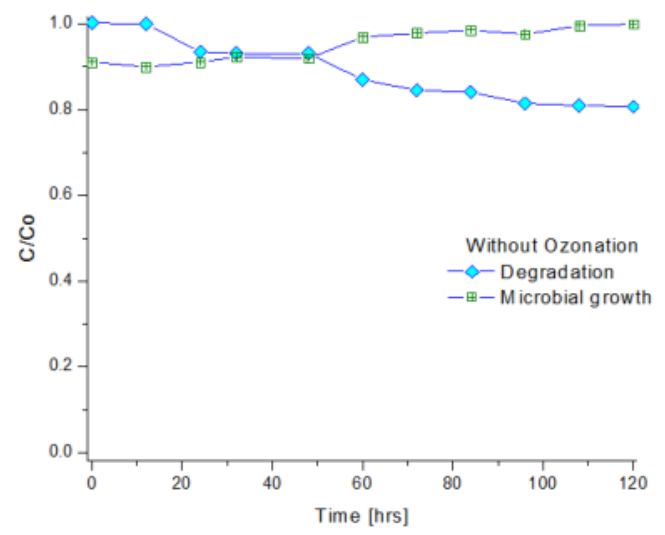

Figure 4. Growth dynamics of biomass and decomposition of organic matter without pre-treatment with ozone.

Nevertheless, microbial growth was obtained but probably because of the consumption of oxalic acid present in non ozonated sample. Taking into account that oxalic acid (which could be eliminated by microorganisms) is in real water (before ozonation) and this compound is formed further in ozonation, it is expected that combined treatment was successful. In general, by comparing in Figure 4 the growth / degradation dynamics appears in normalized form to compare them. Both, the increase of the Optical Density measured at $600 \mathrm{~nm}$ (corresponding to microorganisms grow), and the decrease for the consumption of the source of carbon are shown together.

First 20 minutes are characterized by a partial decomposition of the organic compounds up to $10 \%$. However, after 50 minutes, an additional decomposition around $10 \%$ is obtained but no more pollutants elimination was achieved until the end of the experiment (120 hours).

As it was expected, the organic matter decomposition is not significant. Only $20 \%$ of the total organic matter was eliminated due to the complexity and heterogeneity of the wastewater sample. As expected, microbial growth was not considerable because the pollutants nature as well as their concentration in the sample. 


\subsection{Combined treatment}

\subsubsection{Biological treatment of model solution after ozonation step}

Taking into account the results obtained in previous sections, several ozonation conditions were chosen to test combined treatment. Three principal aspects were considered to choose pre ozonation conditions: the reduction (or complete elimination) of CPhs concentration, the accumulation of phenolic compounds and the final production of organic acids. As phenolic compounds are formed since the beginning but rapidly decomposed during ozonation, its presence was evaluated during biodegradation, in order to consider the needy to continue ozonation till remove them. Table 2 shows these pre ozonation conditions with corresponding ozonation products concentration.

\begin{tabular}{|c|c|c|c|c|c|c|c|}
\hline \multirow{6}{*}{ Identified Compounds } & \multicolumn{7}{|c|}{ Concentration (mg/L) } \\
\hline & \multicolumn{7}{|c|}{ Ozonation time (min) } \\
\hline & \multicolumn{4}{|c|}{ 4-CPh } & \multicolumn{3}{|c|}{ 2,4-DCPh } \\
\hline & \multirow{2}{*}{\multicolumn{3}{|c|}{$\begin{array}{c}\mathrm{pH} \\
7\end{array}$}} & \multirow{2}{*}{ pH 12} & \multirow{2}{*}{\multicolumn{2}{|c|}{$\begin{array}{c}\mathrm{pH} \\
7\end{array}$}} & \multirow{3}{*}{$\begin{array}{c}\mathrm{pH} \\
12 \\
5\end{array}$} \\
\hline & & & & & & & \\
\hline & 10 & 15 & 30 & 5 & 5 & 8 & \\
\hline 4-CPh & 9 & - & - & 18 & - & - & - \\
\hline 2,4-DCPh & - & - & - & - & 18 & - & - \\
\hline Oxalic Acid & 10 & 15 & 27 & 30 & 6 & 9 & 48 \\
\hline Formic Acid & 154 & 137 & 43 & 39 & 36 & 50 & 54 \\
\hline
\end{tabular}

Table 2. Ozonation conditions for the sequential treatment

In order to measure the biodegradation of pre-ozonated samples, the total area registered in HPLC chromatograms (under each condition reported in Table 2) was integrated. A normalized version of this area was used to evaluate the evolution of pollutants decomposition (before biodegradation). For the model sample, total degradation of two kinds of substrates (phenolic compounds and organic acids) was monitored. We could corroborate that the amount of each substrate (phenolics or organic acids) accumulated in ozonation has a very important influence during biodegradation step.

Figures 5, 6, 7 and 8 show the biodegradation on combined treatment of organic acids and phenolic compounds. For both kinds of substrates, during the first two days of biodegradation, it is remarkable the influence of ozonation conditions (Table 2). Indeed, it is a direct relationship between the formation-decomposition dynamics of by-products ozonation and the biodegradation facility of different kind of substrates.

Remembering the separated processes describe above, during ozonation, the phenolic compounds tend to accumulate during the first stage of treatment and after that, they tend to decomposed (Figures 5 and 6 for 4-CPh and 2,4-DCPh respectively). On the other hand, organic 
acids are formed since the beginning and they tend to accumulate (being the oxalic acid the most representative acid at the end of ozonation). On the other hand during biodegradation the oxalic and formic acids demonstrate to be easier to eliminate than phenolic compounds (Figures 7 and 8 for 4-CPh and 2,4-DCPh respectively). Under this perspective, it is very clear why during the first days of biodegradation in combined treatment; the phenolic compounds are more degraded if the ozonation time was higher (under $\mathrm{pH} 7$ ). Without a doubt, ozonation partially degraded these compounds, during biodegradation and then, they are less complicated to be eliminated by microorganisms. This last idea is confirmed by the decomposition dynamics observed for biodegradation of 4-CPh and 2,4-DCPh ozonated under $\mathrm{pH} 12$. Similar behavior was observed for organic acids biodegradation.

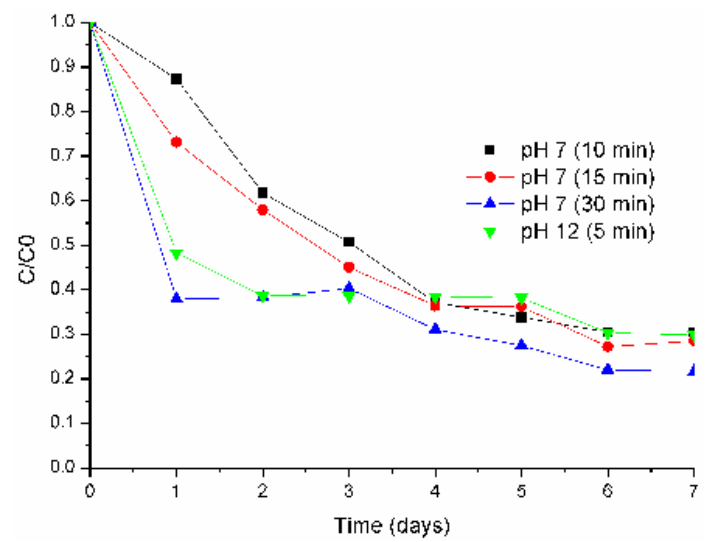

Figure 5. Biodegradation of phenolic compounds accumulated during 4-CPh ozonation

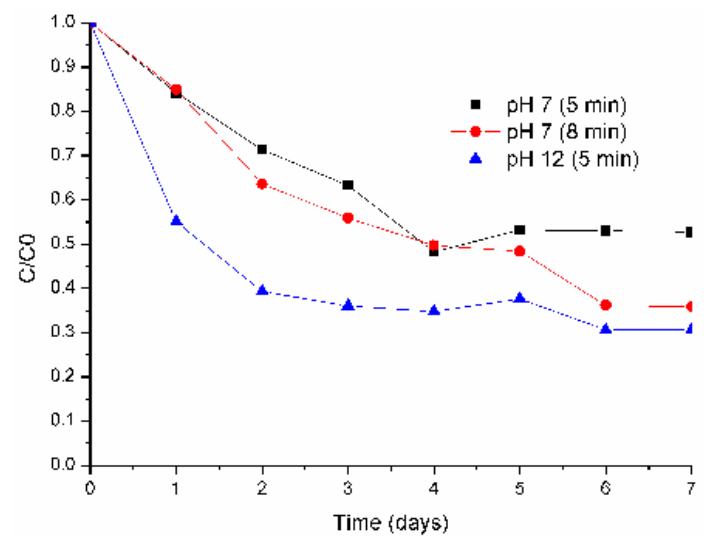

Figure 6. Biodegradation of phenolic compounds accumulated during 2,4-DCPh ozonation 


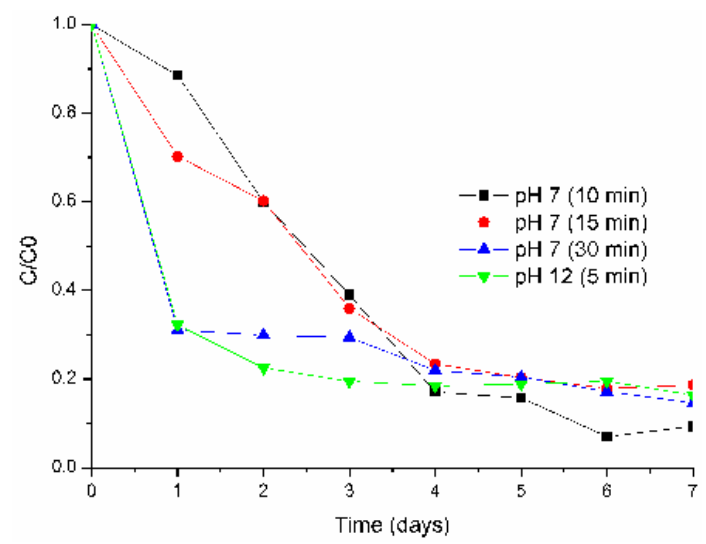

Figure 7. Biodegradation of organic acids accumulated during 4-CPh ozonation

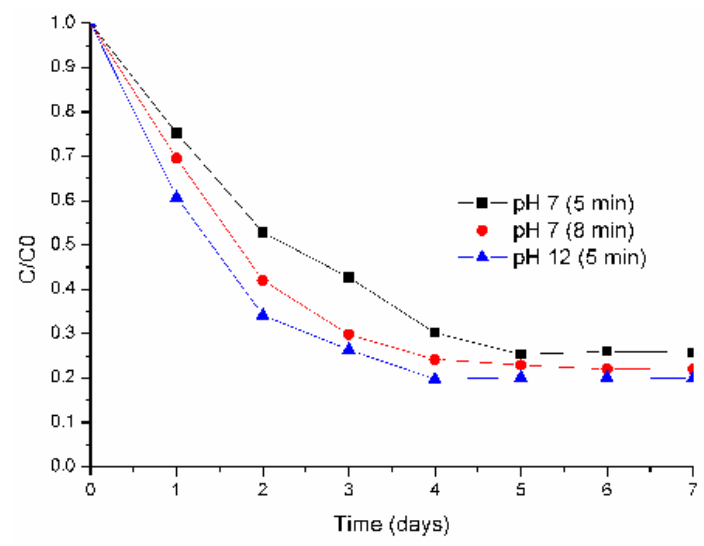

Figure 8. Biodegradation of organic acids accumulated during 2,4-DCPh ozonation

Biodegradation profiles are similar for phenolic compounds and organic acids. These organic acids are easier to eliminate than phenolic compounds. The 4-CPh decomposition degree for each substrate was between $81-90 \%$ and $70-78 \%$ for organic acids and phenolic compounds, respectively. On the other hand, 2,4-DCPh have decomposition degree between $74-80 \%$ and $47-69 \%$ for organic acids and phenolic compounds correspondingly. Table 3 shows a summary of elimination efficiency obtained for each pre ozonation condition.

It is important to notice that no matter the ozonation conditions, resulting by-products (phenolic compounds as well as organic acids) were metabolized by the microbial population since the beginning of the biotreatment, some of them faster than the others, but thank to 


\begin{tabular}{|c|c|c|c|c|}
\hline \multirow{2}{*}{ Compound } & \multirow{2}{*}{$\mathrm{pH}$} & \multirow{2}{*}{ Ozonation time (min) } & \multicolumn{2}{|c|}{ \% Biodegradation } \\
\hline & & & Organic Acids & Phenolic Compounds \\
\hline \multirow{4}{*}{$4-\mathrm{CPh}$} & \multirow{3}{*}{7} & 10 & 90 & 70 \\
\hline & & 15 & 81 & 71 \\
\hline & & 30 & 85 & 78 \\
\hline & 12 & 5 & 86 & 70 \\
\hline \multirow{3}{*}{ 2,4-DCPh } & \multirow{2}{*}{7} & 5 & 74 & 47 \\
\hline & & 8 & 78 & 64 \\
\hline & 12 & 5 & 80 & 69 \\
\hline
\end{tabular}

Table 3. Percentage biodegradation of 4-CPh and 2,4-DCPh pre-ozonated.

previous acclimation the microbial consortia is able to consume ozonated substrates. It is remarkable that residual 4- $\mathrm{CPh}$ (not fully eliminated during ozonation) remaining in ozonated samples during 10 and 5 minutes under pH 7 and 12, respectively was eliminated in biodegradation during the first day of treatment.

Analyzing the individual biodegradation profile of each compound formed during previous ozonation, a serial degradation is inferred. This means than some compounds were preferably consumed (in the earlier days of biodegradation) because of their energetic wealth or ease of degradation. When those organics were depleted, microbial consortium was able to metabolize all others (data not shown).

As it was previously mentioned, a microbial consortium was acclimated to specific carbon source, in this case, ozonation by-products. So, microbial population had developed specific abilities to degrade ozonation products.

Figure 9 shows the global biodegradation behavior in combined treatment for 4-CPh ozonated 10 minutes at $\mathrm{pH}$ 7. Substrates elimination and microbial growth are parallel. They were presented in a normalized way as diminution of the HPLC area (phenolic compounds and organic acids) and optical density increase.

The correlation between the optical density measured at $600 \mathrm{~nm}$ and the pollutants decomposition suggests organic matter integration in the biomass concentration. Between the day 0 and 1, poor degradation is obtained and in agreement, poor microbial growth. Between day 1 and 4, the major organic acids depletion and an important one for phenolic compounds is observed, so a second growth step appears as a result of metabolism of these substrates. Between day 4 and 7 the third growth step is observed as a result of metabolism of residual substrates (phenolic compounds and organic acids consumed until the end). It is remarkable that biodegradation trends of both substrates are simultaneous. However, from the second day, consortium shows an evident preference for organic acids ( $90 \%$ removal) over the phenolic compounds (70\% elimination). Those dynamics were similar for all the combined treatments considered in this study (data not shown). 


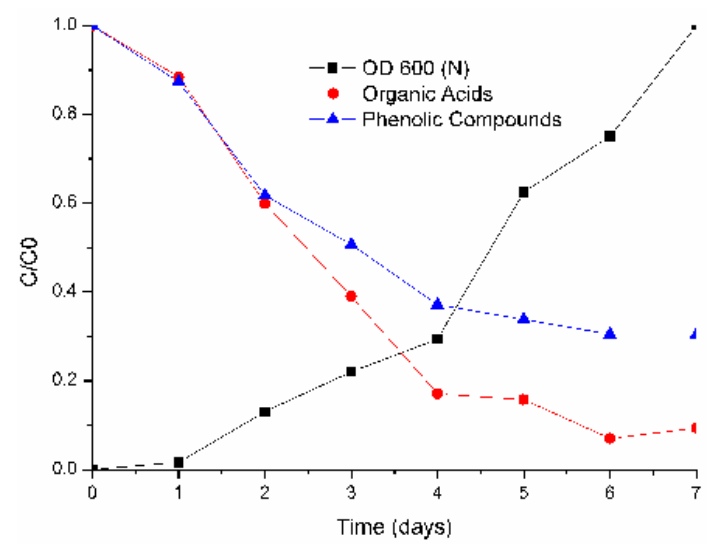

Figure 9. Substrates degradation and microbial growth during biodegradation of pre-ozonated 4-CPh ( $\mathrm{pH} \mathrm{7,10} \mathrm{min).}$

Microbial growth in ozonated substrates was followed by optical density at $600 \mathrm{~nm}$. This analysis was done when different pre-ozonation times (10,15 and 30 minutes for $\mathrm{pH}$ is 7.0 and 5 minutes for $\mathrm{pH}$ is fixed to 12.0) were considered.

When 4-CPh is ozonated, microbial growth was faster when $\mathrm{pH}$ is 7.0 and ozonation time is fixed to 30 minutes. This accelerated biomass accumulation is associated to the major pollutants decomposition. Moreover, when $\mathrm{pH}$ was fixed to 12 and the reaction time was 5 minutes, the lower biomass velocity growing was achieved. If $\mathrm{pH}$ was fixed to 7.0 , when reaction time was 15 minutes, the lag phase was delayed more than any others (Figure 10). This is explained by the accumulation of phenolic compounds under this reaction conditions. This is confirmed by the faster biomass accumulation when ozonation time was 10 minutes and phenolic compounds were not so higher than the previous case.

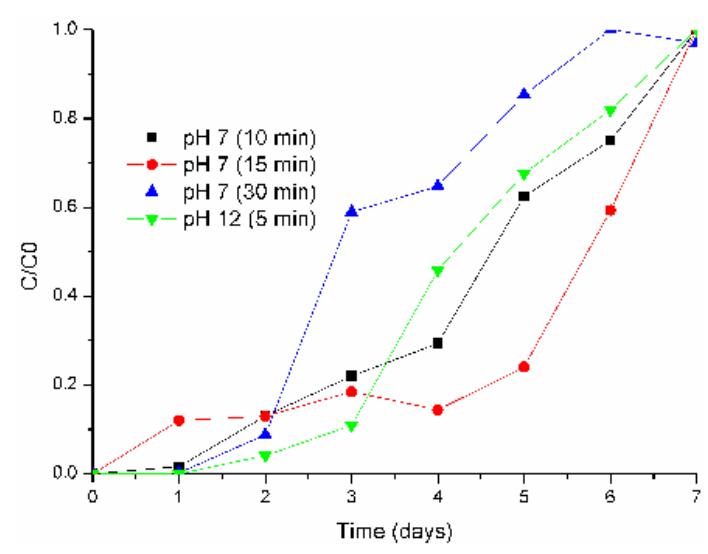

Figure 10. Biomass growth in biodegradation of organic acids accumulated during 4-CPh ozonation 
When 2,4-DCPh was ozonated, the faster biomass accumulation was obtained when $\mathrm{pH}$ was 7.0 and the ozonation time was 8.0 minutes. Once again this condition coincides to the case when lower phenolic derivatives were observed in the reactor. Indeed, when $\mathrm{pH}$ was 7.0 with reaction time was 5 minutes and when $\mathrm{pH}$ was 12 and reaction time was 5 , a lower biomass accumulation was determined. This is in agreement to the previous case, because under these two cases higher phenolic concentrations were observed. As one can understand, when phenolic compounds were at these levels, no important organic acids were in the reactor. This is a contrary situation to the case when phenolics compounds were at their minimum (among other studied cases) concentrations.

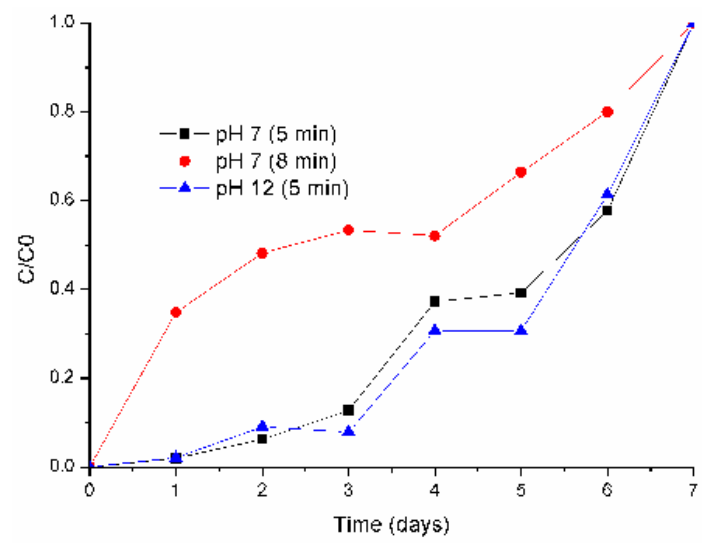

Figure 11. Biomass growth in biodegradation of phenolic compounds accumulated during 2,4-DCPh ozonation

Finally, Figures 12 and 13 show the UV spectra obtained after the combined treatment. These treatments were developed according to conditions presented in table 2.

Major elimination by combined treatment was obtained when most of the not identified compounds were decomposed, respectively (4-CPh ozonated during $30 \mathrm{~min}$ at $\mathrm{pH} 7$ ). This result is in agreement to the higher biomass accumulation observed in Figure 10.

Pre-ozonation conditions had an important influence on the overall degradation. When most of the no identified compounds were decomposed during ozonation (in the subsequent biodegradation step) the UV spectra is very close to the control case (mineral media, absence of contaminant).

It can be noticed that ozonation by-products were not as toxic as the original ones. This is explained because they were simultaneous consumed and corresponding to the microbial growth. In all the studied combined treatments, organic acids were the preferred substrates, 


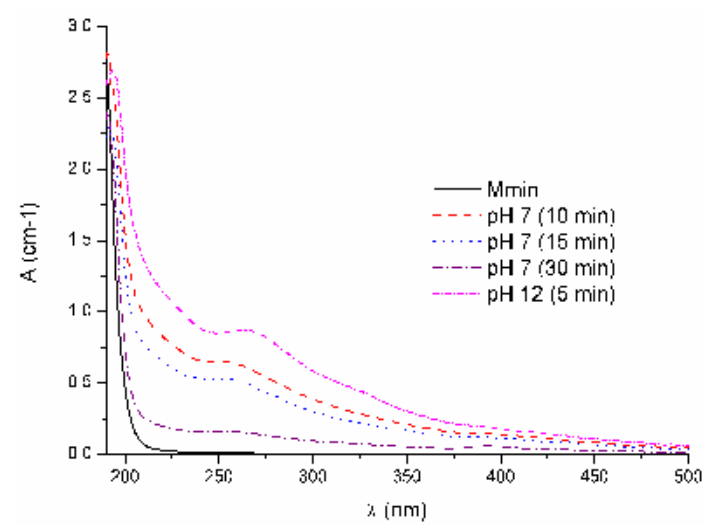

Figure 12. Effect of combined treatment in UV spectra of 4-CPh.

as they were assimilated faster than phenolic compounds. Indeed, phenolic derivatives have shown to serve as inhibitors of the biomass growing.

When 2,4-DCPh was ozonated, a similar condition to the previous one is recognized. The correlation between the biomass growing (showed in the Figure 11) and the phenolics concentrations was confirmed. Moreover, if $\mathrm{pH}$ was fixed to 7 and ozonation time was 8 minutes, an important organic matter decrease was observed (Figure 13).

A slighty difference between this case and the previous one shoukd be remarked: the higher organic matter decomposition is gotten. This is explained by the reaction mechanism that has been identified in preliminar studies. In this case, toxicity of byproducts can have a remarkable role on the biomass growing.

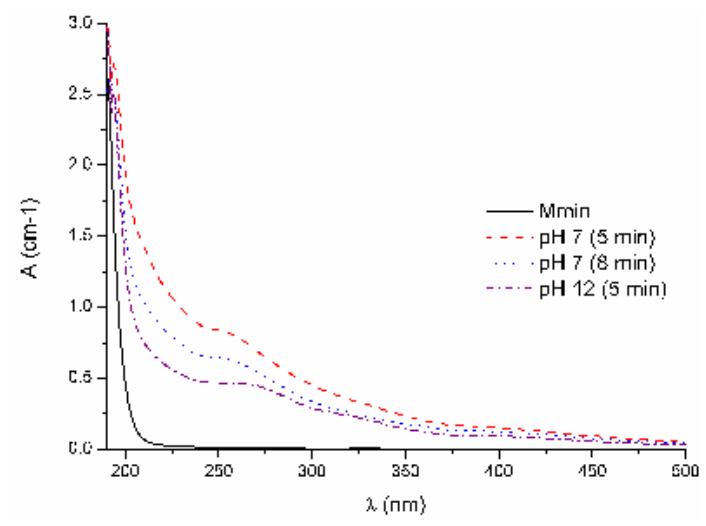

Figure 13. Effect of combined treatment in UV spectra 2,4-DCPh. 


\subsubsection{Biological treatment of real residual water after ozonation step}

Two important aspects were observed to improve biodegradation in combined treatment of model solution: 1) phenolic compounds must be eliminated to the lowest achievable value and 2) short chain organic acids should be accumulated as much as possible. Considering these facts and the real residual water ozonation study, two ozonation conditions were chosen to test the suggested combined treatment: 30 and 60 minutes under $\mathrm{pH}$ 7. These conditions were selected from the study regarding model sample.

Figures 14 and 15 showed the global biodegradation behavior in combined treatment for real water ozonated during 30 and 60 minutes. In a similar fashion to the model solution, substrate elimination and microbial growth are parallel. To compare the results obtained for the model sample and the real wastewater, biomass growing and substrate are presented in normalized way. They are presented as diminution of UV spectra signal and optical density increases correspondingly.



Figure 14. Growth dynamics of biomass and decomposition of organic matter after 30 minutes of ozonation.

During the first 12 hours of biodegradation for real water previously ozonated 30 minutes, more than $60 \%$ of organic matter has been metabolized by microorganisms. Moreover, after 5 days, $82 \%$ of the initial substrate was removed. In the same way, to the real water, a second sample was ozonated 60 minutes. During the first 12 hours, $78 \%$ of the substrate was eliminated and after 5 days, $83 \%$ of substrate was metabolized. As it can be observed, the degradation of all compounds that started from the first minutes. 


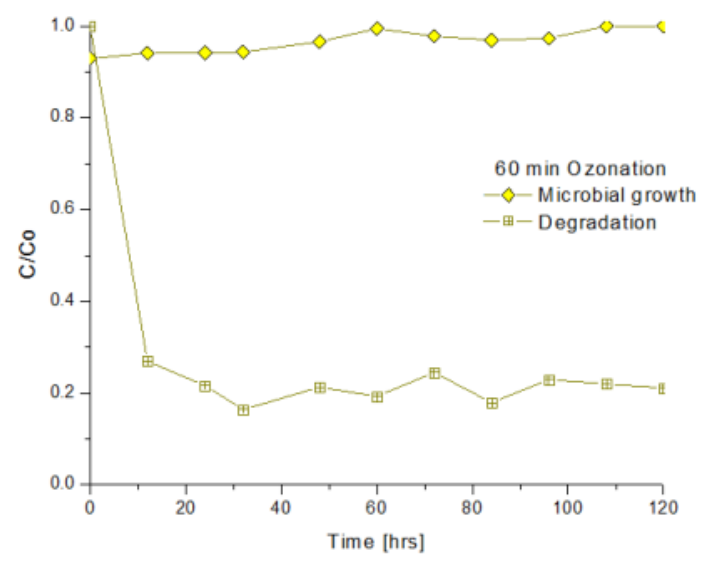

Figure 15. Growth dynamics of biomass and decomposition of organic matter after 60 minutes of ozonation.

This behavior is explained by transformation of pollutants performed by microorganisms. In particular, less toxic substrate as short organic acids were again preferred by them. A decomposition degree of $72 \%$, after 120 hours was gotten.

In the same way for the sample ozonated by 60 minutes, after the first 12 hours, initial substrate was eliminated $78 \%$. When the biodegradation was stopped, an organic matter decrease of $83 \%$ was obtained. On the other hand, it is necessary to pay special attention to the almost unchangeable behavior after 30 hours. This is attributed to the formation of some specific products of biodegradation, which can perhaps be subsequently consumed by microorganisms (tendency to continue decreasing). Remembering that most of the ozonated real water is composed of oxalic acid and similar short chain acids. Therefore, the substrate consumption in real water samples is having a similar portrait to organic acids consumption in model solution, it means, since the biodegradation begins, all these acids are metabolized.

Figure 16 shows the dynamics of the three systems after 5 days of biodegradation. This time was selected as the maximum time for the bioprocess. This study presented a removal of organics of $85 \%$ for sample ozonated by 30 minutes and $89 \%$ for the sample ozonated by 60 minutes.

Finally, in a quantitative way the global effect of biodegradation is the elimination of oxalic acid that was previously formed during ozonation. It is clearly observed that there is a decrease in the concentration of oxalic acid during biodegradation.

In the same way, 30 minutes of ozonation as pre-treatment is more efficient than the other one. They both have similar effects but the suggested one is using half of time for treatment with ozone. As result, final costs of combined treatment are reduced. 


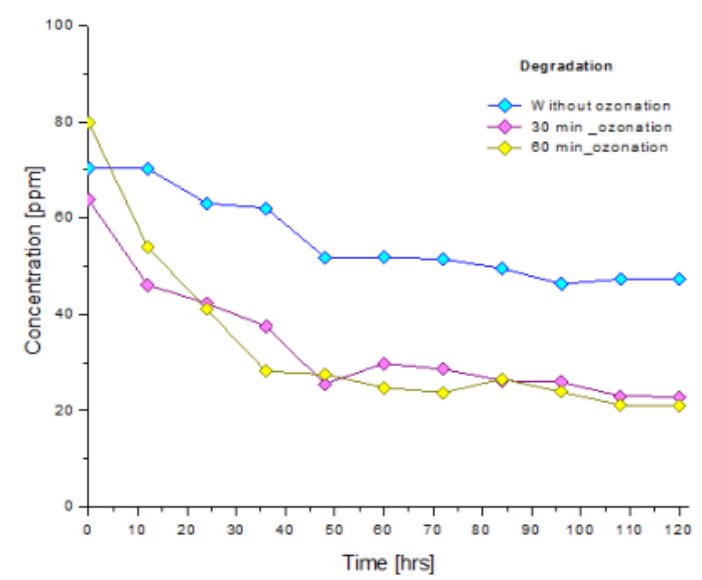

Figure 16. Decomposition dynamics of the three systems (without ozonation, 30 and 60 min of ozonation) after 5 days of biodegradation.

\section{Conclusions}

The combine residual water treatment using ozone before biodegradation seems to be an interesting option to eliminate more complex and toxic contaminants. The combined treatment may handle the aforementioned type of organics mixtures but with less cost than the pure chemical method and with a shorter treatment period than the biological procedure. For the model residual water, the preliminary ozonation decompose organics in the complex mixture and produce more biodegradable species like organic acids. Longer ozonation times are better if one takes into account the decomposition and accumulation dynamics of both, phenolic compounds and organic acids. When most of the no identified compounds were decomposed during ozonation, after the biodegradation step, the UV spectra was very close to the mineral media one in absence of contaminant. Then a major grade of mineralization after combined treatment is obtained. The previous acclimation of the consortium also showed an improvement of the complete treatment scheme for model residual water. The mineralization of ozonation by-products was confirmed by the microbial growth (in aerobic biodegradation, microbial growth is always accompanied by $\mathrm{CO} 2$ production). In the real residual water, the obtained results confirmed the completely decomposition of toxic residues after 60 minutes of ozonation. The decomposition dynamics of lignin derivatives and chlorinated phenols are proportional to the formation of the oxalic acid. This is the main final product of ozonation. Degradation dynamics of these compounds are shown as well as the formation of the oxalic acid. 


\section{Acknowledgements}

This work was supported through funding provided by CONACYT grants 49367, 60976. Also, authors thanks to the IPN project SIP-20120406.

\section{Author details}

P. Guerra ${ }^{1}$, J. Amacosta ${ }^{1}$, T. Poznyak ${ }^{1}$, S. Siles ${ }^{1}$, A. García ${ }^{2}$ and I. Chairez ${ }^{3^{*}}$

*Address all correspondence to: isaac_chairez@yahoo.com

1 Superior School of Chemical Engineering, National Polytechnic Institute (ESIQIE-IPN), Mexico

2 Instituto Tecnológico de Estudios Superiores de Monterrey, Campus Guadalajara (ITESM), Mexico

3 Professional Interdisciplinary Unit of Biotechnology, National Polytechnic Institute (UPIBI-IPN), Mexico

\section{References}

[1] Grau, P. (1991). Textile industry wastewater's treatment. Water Sci. Technol. , 24, 97-103.

[2] Beltrán, F. J, Encinar, J. M, \& González, J. F. (1997). Industrial wastewater advanced oxidation. Ozone combined with hydrogen peroxide or UV radiation, Water Res. , $31,2415-2428$.

[3] Banerjee, A, \& Ghosha, A. K. (2010). Isolation and characterization of hyper phenol tolerant Bacillus sp from oil refinery and exploration sites. J. Hazard. Mater. , 173, 783-788.

[4] Epa, U. S. http://www.epa.gov/waterscience/methods/pollutants.htm, last accessed (2010).

[5] Poznyak, T. I, \& Vivero, J. L. (2005). Degradation of aqueous phenol and chlorinated phenols by ozone. Ozone: Sci. Eng. , 27, 447-458.

[6] Saravanan, P, Pakshirajan, K, \& Saha, P. (2008). Growth kinetics of an indigenous mixed microbial consortium during phenol degradation in a batch reactor. Bioresour. Technol. , 99, 205-209. 
[7] Wei, G, Yu, J, Zhu, Y, Chen, W, \& Wang, L. (2008). Characterization of phenol degradation by Rhizobium sp. CCNWTB 701 isolated from Astragalus chrysopteru in mining tailing region. J. Hazard. Mater. , 151, 111-117.

[8] Abuhamed, T, Bayraktar, E, Mehmetoglu, T, \& Mehmetoglu, U. (2004). Kinetics model for the growth of Pseudomonas putida F1 during benzene, toluene and phenol biodegradation. Process Biochem. , 39, 983-988.

[9] Kumar, A, Kumar, S, \& Kumar, S. (2005). Biodegradation kinetics of phenol and catechol using Pseudomonas putida MTCC 1194. Biochem. Eng. J. , 22, 151-159.

[10] Essam, T, Amin, M. A, Tayeb, O. E, Mattiasson, B, \& Guieysse, B. (2010). Kinetics and metabolic versatility of highly tolerant phenol degrading Alcaligenes strain TW1. J. Hazard. Mater. , 173, 783-788.

[11] Bajaj, M, Gallert, C, \& Winter, J. (2009). Phenol degradation kinetics of an aerobic mixed culture. Bioch. Eng. J. , 46, 205-209.

[12] Nam, K, \& Kukor, J. (2000). Combined ozonation and biodegradation for remediation of mixtures of polycyclic aromatic hydrocarbons in soil. Biodegrad. , 11, 1-9.

[13] Beltran-heredia, J, Torregrosa, J, Dominguez, J. R, \& Garcia, J. (2000). Aerobic biological treatment of black table olive washing wastewaters: effect of an ozonation stage. Process Biochem. , 35, 1183-1190.

[14] Zhao, G, Zhou, L, Li, Y, Liu, X, Ren, X, \& Liu, X. (2009). Enhancement of phenol degradation using immobilized microorganisms and organic modified montmorillonite in a two-phase partitioning bioreactor. J. Hazard. Mater. , 169, 402-410.

[15] Chen, K. C, Lin, Y. H, Chen, W. H, \& Liu, Y. C. (2002). Degradation of phenol by PAA-immobilized Candida tropicalis. Enzyme Microb. Technol. , 31, 490-497.

[16] Hong PKAZeng Y. ((2002). Degradation of pentachlorophenol by ozonation and biodegradability of intermediates. Water Res. , 36, 4243-4254.

[17] Khokhawala, I. M, \& Gogate, P. R. (2010). Degradation of phenol using a combination of ultrasonic and UV irradiations at pilot scale operation. Ultrason. Sonochem. , $17,833-838$.

[18] Chaichanawong, J, Yamamoto, T, \& Ohmori, T. (2010). Enhancement effect of carbon adsorbent on ozonation of aqueous phenol. J. Hazard. Mater. , 175, 673-679.

[19] Derudi, M, Venturini, G, Lombardi, G, Nano, G, \& Rota, R. (2007). Biodegradation combined with ozone for the remediation of contaminated soils. Eur. J. Soil Biol. , 43, 297-303.

[20] Benitez, F J, Real, F. J, Acero, J. L, Garcia, J, \& Sanchez, M. (2003). Kinetics of the ozonation and aerobic biodegradation of wine vinasses in discontinuous and continuous processes. J. Hazard. Mater. B, 101, 203-218. 
[21] Aparicio, M. A, Eiroa, M, Kennes, C, \& Veiga, M. C. (2007). Combined post-ozonation and biological treatment of recalcitrant wastewater from a resin-producing factory. J. Hazar. Mater. , 143, 285-290.

[22] García-peña, E. I, Zarate-segura, P, Guerra-blanco, P, Poznyak, T, \& Chairez, I. (2012). Enhanced Phenol and chlorinated phenols removal by combining ozonation and biodegradation, , 223, 4047-4064.

[23] Nam, K, Rodríguez, W, \& Kukor, J. (2001). Enhanced degradation of polycyclic aromatic hydrocarbons by biodegradation combined with a modified Fenton reaction. Chemosphere , 45, 11-20.

[24] Contreras, S, \& Rodriguez, M. Al Momani F, Sans C, Esplugas S. ((2003). Contribution of the ozonation pre-treatment to the biodegradation of aqueous solutions of 2,4dichlorophenol. Water Res. , 7, 3164-317.

[25] El-Naas, M H, Al-zuhair, S, \& Makhlouf, S. (2010). Batch degradation of phenol in a spouted bed bioreactor system. J. Ind. Eng. Chem. , 16, 267-272.

[26] Adav, S. S, Chen, M Y, Lee, D. J, \& Ren, N. Q. (2007). Degradation phenol by Acinetobacter strain isolated from aerobic granules. Chemosphere , 67, 1566-1572.

[27] Godjevargova, T, Ivanova, D, Aleksieva, Z, \& Dimova, N. (2003). Biodegradation of toxic organic components from industrial phenol producing wastewater by free and immobilized Trichospora cutaneum R 57. Process Biochem. , 38, 915-920.

[28] Edalatmanesh, M, Mehrvar, M, \& Dhib, R. (2008). Optimization of phenol degradation in a combined photochemical-biological wastewater treatment system. Chem. Eng. Res. Des. , 86, 1243-1252.

[29] Dong, Y, Yang, H, He, K, Wu, X, \& Zhang, A. (2008). Catalytic activity and stability of $\mathrm{Y}$ zeolite for phenol degradation in the presence of ozone. Appl. Catal. B: Environ. , $82,163-168$.

[30] Ali, M, \& Sreekrishna, T. R. (2001). Aquatic toxicity from pulp and paper mill effluents: A Review. Advances in Environmental Research , 5, 175-196.

[31] Amat, A. M, Arques, A, Miranda, M. A, \& López, F. (2005). Use of ozone and/or UV in the treatment of effluents from board paper industry. Chemosphere 60 (8), 1111-1117.

[32] Bijan, L, \& Mohseni, M. (2005). Integrated ozone and biotreatment of pulp mill effluent and changes in biodegradability and molecular weight distribution of organic compounds. Water Research , 39, 3763-3772.

[33] Industry Profile sponsored by Contaminated Land and Liabilities Division(1996). Pulp and Paper Manufacturing Works. Department of the Environment Industry Profile, UK 
[34] Fengel, D, \& Wegener, G. Wood: Chemistry, ultraestructure and reactions". Walter de Gruyter ((1984). Berlin/New York.

[35] Freudenberg, K, \& Neish, A. C. (1968). Constitution and Biosynthesis of Lignin. Berlin: Springer-Verlag, 129.

[36] HillCallum A.S. ((2006). Wood modification. Chemical, Thermal and Other Processes. John Wiley \& Sons, Ltd, EEUU

[37] AdlerErich. ((1977). Lignin chemistry-past, present and future. Wood Science and Technology, 11(3), 169-218

[38] Demin, V. A, Shereshovets, V, \& Monakov, J. B. (1999). Reactivity of Lignin and Problems of its Oxidative Destruction with Peroxy Reagents. Russian Chemical Reviews, 68(11), 937-356.

[39] Peng, G, \& Roberts, J. (2000). Solubility And Toxicity Of Resin Acids. Water Research, 34(10), 2779-2785.

[40] RowellRoger. ((2012). Handbook of wood chemistry and wood composites. 2nd Edition. CRC Press Taylor \& Francis Group, EEUU.

[41] Ruiz-dueñas, F. J, \& Martínez, A. T. (2009). Microbial degradation of lignin: how a bulky recalcitrant polymer is efficiently recycled in nature and how we can take advantage of this. Microbial Biotechnology 2(2), 164-177

[42] Buitron, G, \& Gonzalez, A. (1996). Characterization of the microorganisms from an acclimated activated sludge degrading phenolic compounds, Water Sci. Technol. , 34, 289-294.

[43] Kimet., al., ((2002). Biodegradation of phenol and chlorophenols with defined mixed culture in shake-flasks and a packed bed reactor, Process Biochem., , 37, 1367-1373.

[44] Nay, O, Erdeml, E, Kabdali, I, \& Lmez, T. (2008). Advanced treatment by chemical oxidation of pulp and paper effluent from a plant manufacturing hardboard from waste paper. Environmental Technology. , 29, 1045-1051.

[45] García-peña, E. I, Zarate-segura, P, Guerra-blanco, P, Poznyak, T, \& Chairez, I. (2012). Enhanced phenol and chlorinated phenols removal by combining ozonation and biodegradation, Water, Air, and Soil Pollution, 223 (7), 4047-4064.

[46] Poznyak, T. I, \& Vivero, E. J. L. (2005). Degradation of aqueous phenol and chlorinated phenols by ozone, Ozone Science \& Engineering, 27 (6), 447- 458. 
